\title{
SPECIALIZED VISUAL EXPERIENCES
}

\author{
By Casey Landers
}

\begin{abstract}
Through extensive training, experts acquire specialized knowledge and abilities. In this paper, I argue that experts also acquire specialized visual experiences. Specifically, I articulate and defend the account that experts enjoy visual experiences that represent gestalt properties through perceptual learning. I survey an array of empirical studies on face perception and perceptual expertise that support this account. I also look at studies on perceptual adaptation that some might argue present a problem for my account. I show how the data are subject to an interpretation that is friendly to it. Last, I address two theoretical objections to the claim that visual experiences represent gestalt properties.
\end{abstract}

Keywords: visual expertise, visual experience, gestalt property, perceptual learning, rich content.

Experts have specialized knowledge and abilities. This paper concerns whether experts could also have specialized visual experiences. What I have in mind by 'specialized visual experience' is that even though both an expert and a novice receive the same stimulus input and have normally functioning visual systems, the expert enjoys a different visual experience than the novice. I will refer to the thesis that experts enjoy specialized visual experiences as 'Specialized'.

In this paper, I present an account that shows how Specialized could be true. The account comes in two parts. The first is about what kinds of properties specialized visual experiences represent. The second is about how those properties come to be represented. In a nutshell, specialized visual experiences represent gestalt properties through perceptual learning processes. The two parts of the account are not completely novel. Philosophers and Gestalt psychologists first theorized about gestalts over a century ago, and cognitive psychologists have been talking about perceptual learning processes since the mid-twentieth century. What is novel about the account is that no one in the current philosophy of perception literature has put gestalt properties and 
perceptual learning together in the way I do here to make sense of Specialized and visual expertise. ${ }^{1}$

The paper is as follows. Section I makes some preliminary remarks about terms, assumptions, and the background debate. Section II lays out what gestalt properties are. Section III introduces what perceptual learning is, and homes in on unitization and differentiation. Section IV presents some empirical studies on face and expert perception, the results of which are exactly what we would expect to see if my account is correct. I also consider whether certain empirical studies on perceptual adaptation present a problem for my account in Section V. Section VI then presents and responds to two objections to the claim that visual experiences represent gestalt properties rather than subordinate level rich properties. Section VI. I presents and responds to the 'Goldilocks Problem', which is the problem of identifying a gestalt property that is the right degree of abstraction. Section VI.2 discusses the 'No Single Gestalt' problem, which is the worry that there is not a single gestalt property that could indicate certain rich properties.

\section{THE BACKGROUND}

Before presenting the debate in which this paper is situated, I should clarify some assumptions and terms. This paper broadly focuses on the kinds of properties visual experiences represent. To understand what it means to say that visual experiences represent something, I should unpack a few notions. First, I'm assuming a common but not uncontroversial view that visual experience is a representation, a state with intentionality. As a representation, visual experience says something about the way the world is, and what it says is the visual experience's content. Content is given by something like truth or accuracy conditions. On this assumption, content is specified by the ways the environment must be to make what the experience says true or accurate, e.g., if my visual experience says there is an apple on the table, it is saying something accurate and true just in case there really is an apple on the table. One final thing to set the stage: Sometimes philosophers in the debate about what vision represents talk about what visual experience represents, and other times they talk about what the visual system represents. I'm focused solely on

\footnotetext{
${ }^{1}$ Stokes (2018) is the only author in the philosophy of perception literature of which I am aware that makes the case that expert visual experiences represent gestalt properties. However, he focuses solely on art expertise, whereas I am focused on visual expertise in general. Arstila (2016) and Connolly (2014) are the only authors in the philosophical literature that argue that perceptual learning processes lead experts to enjoy different visual experiences than novices. Arstila (2016) also notes that experts might visually experience gestalt properties, but this is merely mentioned as a possibility and is not developed or defended in his paper. More will be said in Section I about Connolly's (2014) account.
} 
visual experience, which is fundamentally a phenomenological category, in the sense that experience is constitutively phenomenally conscious, i.e., necessarily there is something it is like to be in this kind of mental state. While I will not write in terms of what visual systems represent, and will not assume that visual systems and visual experiences have the same contents, it may be the case that my account also generalizes to what visual systems represent.

Now I will briefly sketch out the debate surrounding Specialized. Philosophers of perception vastly disagree about what range of properties visual experience represents. The conservative position is that relatively few kinds of properties are visually experienced, namely 'thin' or 'low-level' properties, like shape, color, texture, motion, and direction. Everyone agrees that at least these low-level properties are visually experienced. But many argue that visual experience represents more than just those properties. Usually philosophers use the terms 'rich' or 'high-level' to refer to properties that are not thin or low-level. ${ }^{2}$ Examples of rich properties that philosophers have argued for are natural kind properties (Siegel 2006, 2010), artifact kind properties (Siegel 2006, 2010), mental state properties (Butterfill 2015; Block 20I4; Helton 2018; Neufeld 2018; Toribio 2018), causal properties (Siegel 2009; Butterfill 2009), aesthetic properties (Stokes 20I4, 2018), moral properties, and affordances (Siegel 2014). I will call this cluster of views that agree that more than just low-level properties are visually experienced, 'Rich Content'.

While many philosophers of perception think Rich Content is true, there is little agreement concerning the range of rich properties that are visually experienced. Block (2014), Byrne in Siegel and Byrne (2016), and Burge (2014), for example, are relatively conservative: they think the only rich properties visual experience plausibly represents are ones that are particularly evolutionarily salient. This points to only a few kinds of rich properties, like facial expression properties, causal properties, and properties related to agency. Others are more moderate by allowing a wider range of rich properties. Mandelbaum (2018), in line with Fodor (1998), argues that vision represents basic level category rich properties, like being a dog or being a tree, but does not represent properties at a more specific level of categorization, namely subordinate level rich properties, like being an Australian Shepherd or being a pine. A few philosophers are very liberal in the range of rich properties they posit as the contents of visual experience (Siewert 1998; Siegel 2006, 2010; Bayne 2009). They allow that virtually all kinds of rich properties can be visually experienced. It is at this juncture where expertise enters the debate about what visual experiences represent.

Siegel $(2006,2010)$ specifically appeals to visual expertise to argue for her liberal Rich Content position. One of the main examples Siegel uses concerns a tree expert and novice looking at the same pine. Intuitively, the tree expert's visual experience feels different than the novice's. A plausible way to explain why their experiences feel different is that they represent different properties.

${ }^{2}$ I will say more about how to define these terms at the end of Section II. 
Siegel argues that the expert's visual experience represents the tree as a subordinate level rich property, namely as a pine tree, whereas the novice's does not. ${ }^{3}$ Her view is that experts gain recognitional capacities for subordinate level rich kind properties, and these recognitional capacities cognitively penetrate expert visual experience, resulting in visual experiences of those subordinate level rich kind properties. Siegel's version of Rich Content therefore gives us one way in which Specialized could be true. ${ }^{4}$ Let's call this view 'Subordinate Level Rich Content' or 'SRC' for short.

SRC is controversial for a few reasons. First, not everyone agrees that subordinate level rich kind properties are visually experienced. Second, SRC seems to give a role to cognitive penetration because the expert's recognitional capacity somehow affects their visual experiences. Broadly speaking, cognitive penetration occurs when cognitive states alter the contents of visual experience. It is hotly debated whether and when cognitive penetration actually occurs. Most alleged cases of cognitive penetration are subject to different interpretations ${ }^{5}$ so SRC inherits the controversy surrounding cognitive penetration. The third reason why SRC is controversial is that we don't need SLC to make sense of phenomenal contrasts between the visual experiences of experts and novices. There are other plausible ways to make sense of those phenomenal contrasts.

A different way to make sense of phenomenal contrasts is to agree that experts represent different properties than novices, but to posit that experts merely visually experience different low-level properties. Connolly (2014) articulates and defends this view. He argues that experts pay attention to different low-level properties than novices because experts have gone through a perceptual learning process known as 'attentional weighting'. Sticking with the tree expert example, tree experts might learn to automatically pay attention to the specific shape and color of a tree's trunk and leaves in order to recognize and identify what kind of tree it is. The expert's visual experience doesn't represent subordinate level rich properties or any rich properties at all, though it does still represent different properties than the novice's.

There is a third way of explaining away phenomenal contrasts between experts and novices that Siegel (2010: Ch. 4) briefly mentions. She entertains the possibility that phenomenal contrasts between expert and novice visual

\footnotetext{
${ }^{3}$ Siegel does not use the term 'subordinate level rich property' to describe the property of being a pine tree. Rather, she uses the term 'kind property'.

${ }_{4}^{4}$ According to the conservative version of Rich Content, experts and novices would both represent evolutionarily salient rich properties like being animate or being angry. According to the moderate version, experts and novices would also represent basic level kind properties like being a dog. Under both of these accounts, experts and novices would visually experience the same rich and low-level properties, so both of these accounts would fail to explain Specialized.

${ }^{5}$ See Macpherson (2012) and Stokes (2014) for arguments that visual experience is cognitively penetrated. See Firestone and Scholl (2016) for a detailed survey and critique of many alleged cases of cognitive penetration, and also Arstila (2016).
} 
experiences are generated by the expert visually experiencing a pine tree gestalt that the novice does not visually experience. This is precisely the account that I develop in this paper. Siegel does not spend much time discussing this alternative, but notes some reasons for why she thinks it won't work. In Section VI.I, I will address the worry she briefly raises - a worry that I'm sure some readers will have as well. For now, I want to say more about how my account relates to Connolly's (2014), and then move on to develop the account in detail.

My account is similar to Connolly's in that we both reject SRC and highlight the role of specific perceptual learning processes in visual expertise. The main difference is that I posit that experts visually experience gestalt properties, while Connolly does not explicitly defend this claim. ${ }^{6}$ Despite this difference, I think our accounts could be conjoined to form a more robust and accurate account of Specialized. In combining our accounts, the result would be that experts enjoy different visual experiences than novices for one of two reasons: either their visual systems have undergone attentional reweighting, leading experts to visually experience different thin properties like shape, color, and texture, that novices do not visually experience; or their visual systems have undergone unitization or differentiation, leading experts to visually experience a gestalt property that the novice does not. This combined account of Specialized is extremely plausible, as it can explain away phenomenal contrasts between expert and novice visual experiences, and has empirical support. Since Connolly's account has already been nicely articulated and defended, the remaining discussion focuses on developing my account and keeps this combined view in the background.

Of course, someone could think that Specialized is false. Denying Specialized amounts to saying experts and novices enjoy the same visual experiences. On this view, the expert's ability to recognize something as a subordinate kind is not reflective of something unique about their visual experience. Rather, on this view, what differs between experts and novices is post-perceptual: experts make different, more fine-tuned, more accurate judgments about the objects of their expertise, but their visual experiences are the same as novices. Against this, I'll present some empirical evidence in Section IV that provides good reason to think changes to one's visual experience do in fact take place when one becomes an expert. And as will become clear, my account nicely predicts this empirical evidence. But first, I will lay out in more detail what my account actually is.

\footnotetext{
${ }^{6}$ If it turns out that gestalt properties are low-level properties, then the account I offer here would be a more specific version of Connolly's. I will discuss whether gestalt properties are rich or low-level in the next section.
} 

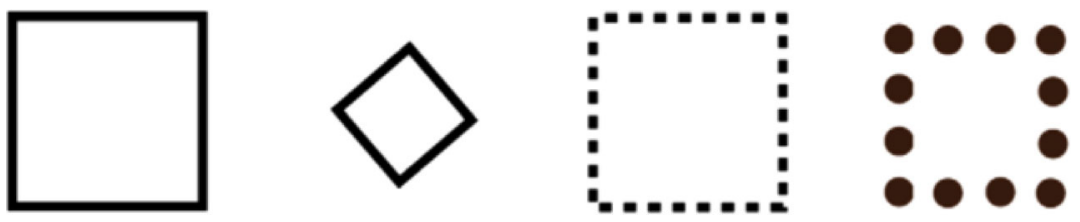

Figure I. Basic shape gestalts. While each of these objects varies in what specific low-level features they instantiate, there is still an abstract structure that we visually experience them as having in common.
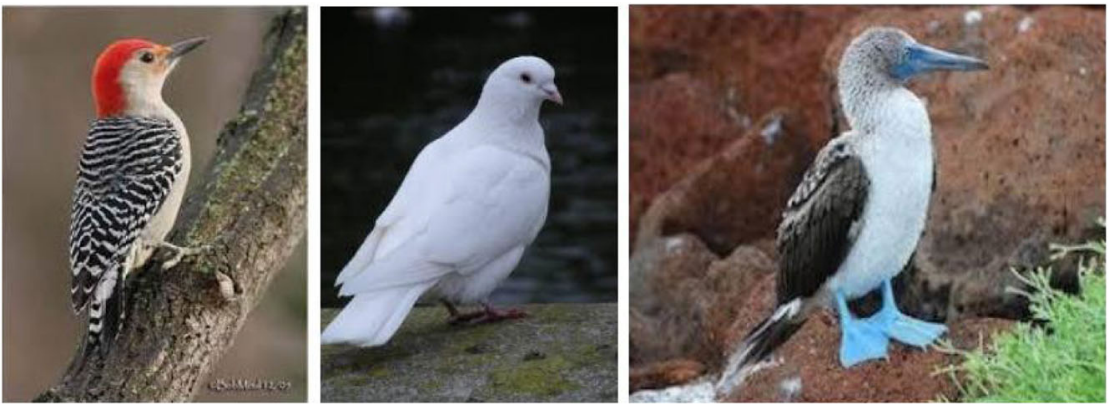

Figure 2. Bird gestalts. The birds pictured here (from left to right: a Red-bellied Woodpecker, a dove, and a Blue Footed Booby) are different in the actual specific low-level properties they each instantiate. Nonetheless, there is an abstract gestalt that is common to all three.

\section{GESTALT PROPERTIES}

My account, which I will simply refer to as 'Gestalt', has two parts. The first part concerns what kinds of properties specialized visual experiences represent, namely gestalt properties, and the second part concerns how those properties come to be represented, namely through perceptual learning. This section describes what I understand gestalt properties to be.

There are a few important features of gestalt properties: they are configural, abstract, and determinable. By 'configural', I mean that a visual gestalt property involves how low-level visual properties are spatially related to each other. Foreground-background relations (e.g., $\mathrm{x}$ in front of y), orientation relations (e.g., x beside/below/above y), and proximity relations (e.g., how close or far $\mathrm{x}$ and $\mathrm{y}$ are from each other) are vital to individuating gestalt properties.

Gestalt properties are also abstract. In Figure I, there are many objects that differ in various ways with respect to which low-level properties they instantiate. Still, there is some abstract and invariant shape that they share. They share a gestalt property. In Figure 2, again, we have three different objects that do not instantiate the exact same low-level properties, yet they share a common gestalt property.

Gestalts are also determinables. There are different determinate ways that a particular kind of gestalt can be realized. There can be slight variations in the specific low-level properties instantiated, while the same abstract determinable 
property is instantiated. Individual members of a kind, like the different squares in Figure I or the individual birds in Figure 2, instantiate different determinates of a gestalt. A helpful example concerns color. The color red is a determinable, while the colors scarlet, maroon, and burgundy are determinates. Scarlet, maroon, and burgundy each are specific ways of being and looking red. In the same way, each of the birds in Figure 2 has low-level properties arranged in a very specific way, and each of those specific low-level arrangements is a way of instantiating a more general bird gestalt. One benefit of thinking of gestalts as determinables is the following: just as we don't think of the color red as merely a disjunction of all of the different determinate red colors, gestalts are also not merely disjunctions of specific arrangements of low-level properties. Rather, the specific arrangements of low-level properties are ways of instantiating the more abstract, determinable gestalt. ${ }^{7}$

Now that I've briefly described what I take gestalt properties to be, one may wonder whether they count as low-level or rich properties. Where exactly do they fit into this distinction? They present a particularly hard case for classification, for the following reasons. The same gestalt can be instantiated with slight variations in low-level configurations. Gestalt properties are a sensory whole that involve abstract configurations of how those low-level properties are spatially related. Thus, gestalt properties may supervene on low-level properties and can be multiply realized, but are not reducible to properties that are uncontroversially categorized as low-level properties. So it doesn't seem like gestalt properties are straightforwardly low-level. This seems like one reason to count them as rich properties. Another reason to count them as rich properties concerns the fact that 'low-level' and 'rich' properties are usually defined ostensively. Rich properties are often defined 'by excluding a small class of [low-level] properties that include color, shape, illumination, and motion' (Siegel 20I0; 99). Indeed, this seems to be the most common way of defining these terms. ${ }^{8}$

However, according to other definitions, gestalt properties would not technically be rich. For example, Siegel also says that low-level properties are those that are 'standardly taken to be... [perceptually] represented' (2010; 7). To my knowledge, no one argues that gestalt properties are not visually experienced or represented. Rather, the controversy surrounding gestalt properties is whether some properties over and above gestalt properties are visually experienced or represented. ${ }^{9}$ This definition makes it sound like gestalt properties

\footnotetext{
${ }^{7}$ I point the reader to Section VI if they have more questions about gestalt properties, like how members of a particular category (Red-Bellied Woodpeckers, for example) could instantiate the same gestalt.

${ }^{8}$ For example, Bayne (2009), Block (2014), Connolly (2014), Helton (2016), Logue (2013), Silins (20II), and Stokes (2018) use this definition.

${ }^{9}$ See Siegel (2010; Chapter 4); Siegel and Byrne (2017); Block (2014); and Burge (2014) for discussions about whether or not more than gestalt properties are visually experienced.
} 
are low-level properties. One might also define rich properties as those that make a significant difference to the amount of beliefs that are perceptually justified. Clearly, if natural kind and artifactual kind properties were the contents of visual experience, a much wider range of beliefs would be perceptually justified than if they were not. On the other hand, it is not clear that gestalt properties as the contents of visual experiences would have the same epistemic effect, which thereby suggests that, again, they should be classified as low-level. One further way to distinguish rich from low-level properties is in neurological terms: low-level properties are the computational outputs of sensory regions in the brain. If gestalt properties are the products of perceptual learning (as I will suggest later in the paper), and since perceptual learning operates within a perceptual system, then gestalt properties would be low-level. Moreover, the representation of gestalt properties does not obviously involve any explicit conceptual labeling, which lends further plausibility to the idea the representation of gestalt properties, like the representation of simple shapes, depends on the processing of visual systems and not on post-perceptual ones.

In short, gestalt properties are not clear cut cases of low-level or rich properties. Three out of the four ways of defining these terms suggest that gestalt properties are low-level. Perhaps one thinks that the distinction between lowlevel and rich isn't metaphysically tractable, and we should use a different way of talking about the contents of perceptual experience. One potentially fruitful idea is that rather than a distinction in kind between low-level and rich properties, these properties are on a spectrum, with gestalt properties somewhere between simple low-level properties, like shape and color, and more rich properties, like natural and artifactual kinds. On this proposal, gestalt properties are 'mid-range' properties: they are over and above paradigmatic low-level properties, but not quite so rich as other properties that are also over and above paradigmatic low-level properties.

Regardless of how we define these terms, it's important to note that even if gestalt properties turn out to be a kind of rich property, they are still alternatives to all other sorts of rich properties in terms of what the contents of visual experience are. The reason why is that anytime someone argues that visual experience represents a basic or subordinate level rich property like a natural or artifactual kind, we can always ask whether visual experience represents a gestalt property instead. In a way, then, whether gestalt properties turn out to be rich properties is slightly orthogonal if we are mainly concerned with the different ways to make sense of how there could be specialized visual experiences.

Neither Block nor Burge use the term 'gestalt'. Block uses the term 'recognitionally co-extensive complexes of low-level properties', while Burge uses the terms 'generic' and 'specific' 'low-level attributives'. Block, Byrne, and Burge think that, save cases where some rich property is particularly evolutionarily salient, properties over and above gestalt properties are not visually represented. 
Before turning to the perceptual learning part of the account, I want to address a lingering worry the reader may have. Experts seem to visually recognize subordinate level rich properties, e.g., the tree expert effortlessly recognizes the pine tree as a pine tree. If gestalt properties are supposed to be alternatives to subordinate level rich properties in terms of the contents of expert visual experiences, the reader may wonder how an expert could effortlessly recognize subordinate level rich properties without visually experiencing those kinds of properties. One of the key ideas behind Gestalt is that expert visual experiences represent gestalt properties that indicate subordinate level rich properties. Experts still recognize subordinate level rich properties because the gestalt properties that they visually experience indicate those rich properties. What do I mean by 'indication'? On a simple analysis, $\mathrm{X}$ indicates $\mathrm{Y}$ when there is a consistent, reliable, contingent connection between $\mathrm{X}$ and $\mathrm{Y}$, such that we would be justified in inferring that $\mathrm{Y}$ is present when $\mathrm{X}$ is and vice versa. For example, the duck gestalt-a complex, abstract, indeterminate shape that ducks generally share-indicates the natural kind property of being a duck, because usually ducks share this duck gestalt, and most things that are this gestalt shape are ducks. This indication is contingent because in other possible worlds, ducks may not have the same appearance as they do in this one, so the gestalt property that indicates ducks in another possible world could be a different one than the gestalt property that indicates ducks in this world. If gestalt properties can indicate subordinate rich kind properties in the way I've briefly sketched, then psychologically speaking, a quick unconscious inference or association between a visual experience of a particular gestalt property can lead to the recognition that a particular subordinate rich property is instantiated. This briefly shows how experts could effortlessly recognize subordinate level rich properties without visually experiencing them. ${ }^{10,11}$

Another worry the reader may have is that while it's coherent that experts visually experience gestalt properties, I have yet to provide any reason to think that they actually do. To this point, I'll present some empirical evidence in Section V that my account nicely predicts. Then in Section VI, I'll respond to worries about whether gestalt properties, in addition to low-level properties, are sufficient to posit as the contents of expert visual experiences. For now, I want to move on to laying out what perceptual learning is, and how perceptual learning seems to be the right kind of process to explain how experts come to visually experience gestalt properties.

\footnotetext{
${ }^{10}$ Burge (2014) also theorizes that rich properties are not visually experienced but are indicated by low-level properties, though he doesn't cash out the notion of 'indication' in any detail.

${ }^{11}$ It is interesting to note the following: those who deny a Liberal form of Rich Content will run into this same problem, including other Rich Content theorists. The Conservative and Moderate Rich Content theorists will deny that a tree expert visually experiences the property of being a pine tree or that the radiologist visually experiences the property of being a tumor. But, presumably, they do not deny that these experts recognize those rich properties.
} 


\section{PERCEPTUAL LEARNING}

In this section, I will briefly introduce what perceptual learning is. Then I will delve into a more in-depth discussion of the particular types that play a role in Gestalt.

Perceptual learning is a process by which an individual learns over a period of time to extract task-relevant information contained in a stimulus. It consists in long-term changes to the perceptual processing of a stimulus due to training and practice (Gibson I963). For some change in recognitional capacities to count as perceptual learning, the change must meet three conditions: I) it is not a short-lived change; 2) it is genuinely perceptual rather than something due to learned inference; and 3) it is a result of training and practice rather than maturation (Connolly 20I7, 2019). Perceptual learning processes are often divided into four different kinds: differentiation, unitization, stimulus imprinting, and attentional weighting. For the sake of this discussion, I will only focus on unitization and differentiation, though I direct the reader to Connolly (2017) for an in-depth survey of all four.

Unitization involves conjoining two separate properties into one perceived property, e.g., when learning to read, you learned to perceptually group letters into discrete units (words), rather than merely as a string of letters. Goldstone and Byrge (2015) describe a helpful way to think about unitization, namely that it is comparable to a memory-based phenomenon known as 'chunking'. Chunking information into discrete semantic units makes it significantly easier to store a large amount of information in working memory. Working memory has a limited storage capacity of about $7+/-2$ items at any given time. However, these items are more like file folders than they are like pieces of information, as we can put more information into each individual item through chunking. For example, remembering a long string of letters like 'S U N M O N T U E S J A N F E B M A R' is easier than 'S O E N T W O G T I C Y C B A M P F'. Even though both contain eighteen letters, the letters in the former string are chunked according to something meaningful (the first three letters of the first 3 days of the week and first 3 months of the year) whereas the latter is a random string of letters. Chunking allows us to manipulate the storage capacity by treating separate bits of information as part of one unit.

A nice example of chunking comes from chess masters who exhibit an enhanced ability to remember a large number of familiar configurations of chess pieces on the board. Based on a series of studies, Gobet and Simon (I996, 2000) suggest that these familiar configurations are stored as chunks and templates. Chess masters do not remember just individual features, like the shape of the rook or the castle, or where the rook or castle are, but must also remember how these shapes are related to each other on the board. After extensive training, what was once represented individually, e.g, each chess piece, or each shape of the chess piece, on different black and red squares, 
comes to be represented as part of a whole, a template of the board. Individual shapes of the chess pieces and their orientations with respect to one another on the chessboard are represented as a unit. This chunk can then facilitate quick, efficient memory retrieval.

Unitization is the perceptual equivalent of chunking. As Goldstone and Byrge (2015) say,

... [O]ur perceptual systems can build new perceptual chunks to encode as single units what would otherwise be complex visual patterns. Whereas for chunks in memory elements are combined together because they make semantic sense, perceptual units are formed because they can be seen as coherent perceptual objects, obeying, for example, the Gestalt laws of proximity, continuity, and closure.

The perceptual units Goldstone and Byrge (2015) speak of are what I have in mind with gestalt properties. They write that there is empirical evidence of unitization for a wide range of objects: birds, words, grids of lines, random wire structures, fingerprints, artificial blobs, and Greebles, which I will discuss shortly (2015: 823).

Unitization as a learning process can potentially lead to a visual experience of gestalt properties. Low-level properties are the basic building blocks for gestalt properties, and unitization is the kind of process that can organize these building blocks into different abstract templates such that the result is a gestalt property that one visually experiences. Unitization can bring out relations between low-level properties as something over and above the lowlevel properties themselves. Instead of simply experiencing an array of shapes, colors, textures, etc., one experiences these low-level properties as conjoined into one coherent unit based on relations that hold between those low-level properties.

Differentiation also seems to play an important, perhaps even an essential, role in visual expertise. This is because differentiation essentially involves telling the difference between two things that one previously did not differentiate. In becoming a dog expert, for example, one learns to perceptually differentiate individual dogs of the same breed from each other. At first, it is quite difficult to tell the dogs apart, but after some time, one can differentiate individuals. Similarly, radiologists must learn to tell the difference between normal tissue and abnormal tissue; bird experts must learn to tell different species from one another; chess masters must differentiate different templates for modes of attack and defense, etc.. Arguably, any form of visual expertise involves differentiating things that novices generally fail to differentiate.

How could differentiation result in an expert's visual experience representing a gestalt property that it did not previously represent? Initially, one might just represent a gestalt property for a basic level category, like a bird or a dog gestalt. Then, with sufficient exposure and certain task demands, the visual system becomes sensitive to slight differences in the configurations that 
subordinate level kinds generally share, and the expert's visual experience comes to represent two different, more specific gestalts. In this way, differentiation could lead to the visual experience and representation of new perceptual units.

Now the basics of my Gestalt account have been laid out. I've presented what exactly gestalt properties are, what perceptual learning is, and how gestalt properties could be the products of perceptual learning processes, specifically unitization and differentiation. The total proposal, then, is that Specialized is true because expert visual systems underwent perceptual learning processes, which lead to the visual representation and experience of gestalt properties that were not represented before. These gestalts indicate subordinate level rich properties that fall under the expert's area of expertise.

\section{BEHAVIORAL STUDIES ON FACE AND EXPERT PERGEPTION}

There is an array of empirical evidence that expert visual experiences represent gestalt properties. ${ }^{12}$ In this section, I will survey two studies on face perception that show humans are visually sensitive to configurations of facial features in detecting faces, which suggests that humans visually experience face gestalts. After looking at two different tasks used in the face perception literature, I will also present studies that suggest visual sensitivity to configurations of low-level features is not unique to faces, but arises with expertise in a particular field. Such evidence is exactly what we should expect if my account of specialized visual experiences is correct.

The two tasks used in behavioral studies that suggest face perception is sensitive to gestalt properties are composite and whole-part tasks. Composite tasks involve conjoining top and bottom halves of different faces together. Young, Hellawell and Hay (I987) asked subjects to identify whether the top or bottom half of a face belonged to a face with which they had been previously familiarized. To adequately do this, subjects had to ignore one half of the face. When the bottom and top parts of the face were aligned, subjects performed poorly. This effect disappeared when the two halves were misaligned. (See Figure 3.) One plausible interpretation of this result is that a whole new face gestalt results from conjoining the bottom and top halves of different faces, which explains why it is difficult to recognize the bottom or top half as belonging to a different face when the two halves are conjoined.

Whole-part tasks also indicate that facial processing is heavily dependent on picking up a face gestalt. If a feature (like a mouth or nose) is processed

${ }^{12}$ The discussion in the psychological literature rarely uses the term 'gestalt property'. Rather, psychologists often use terms like 'holistic processing', 'holistic representation', and 'configural representation'. 


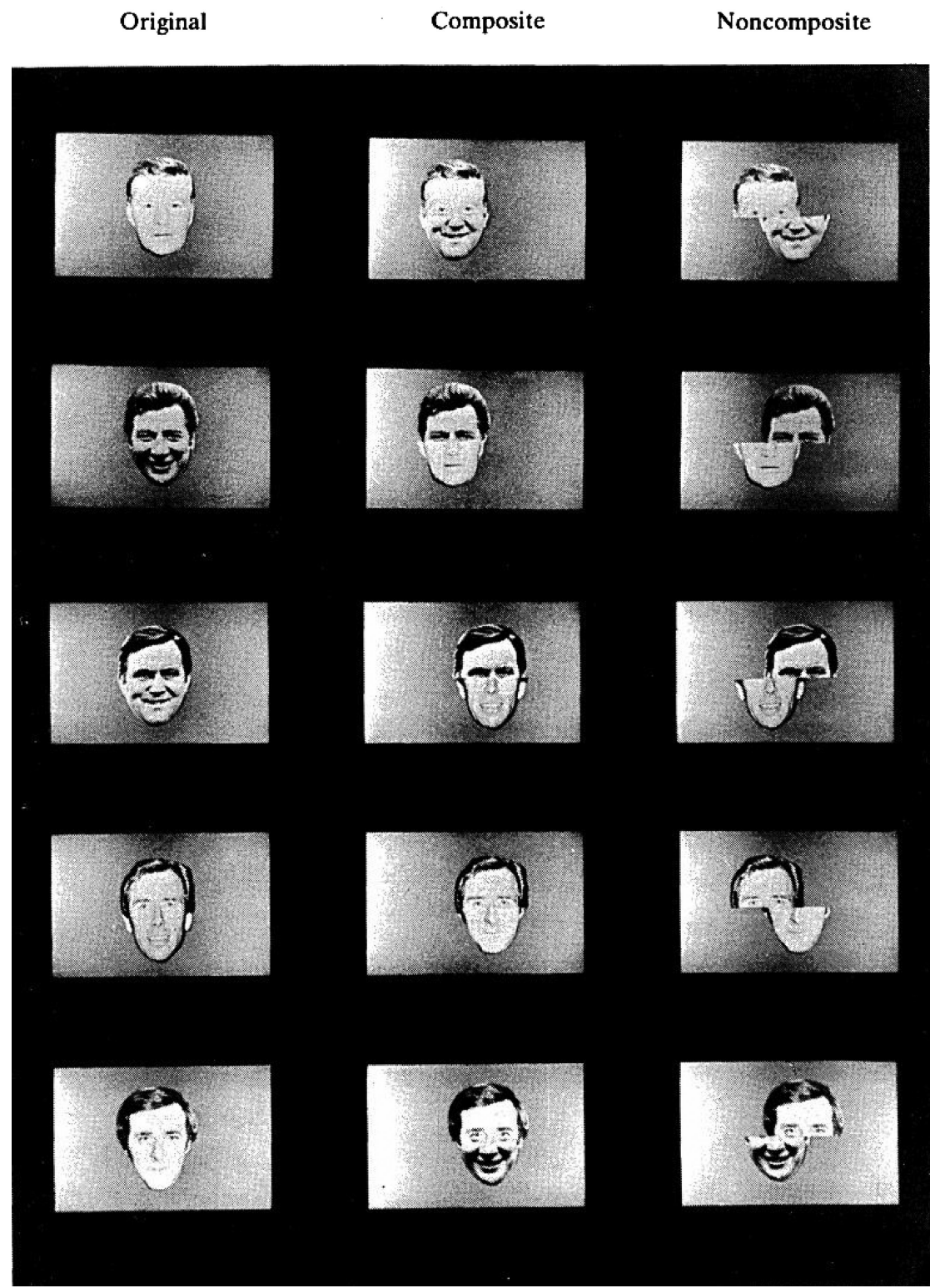

Figure 3. The original stimuli used in Young, Hellawell and Hay's (1987) composite tasks. 
as part of a face gestalt, recognizing the feature in isolation or in a different context (like a different face) should be more difficult than recognizing the feature as part of the gestalt as a whole. Tanaka and Farah (I993) provide confirmatory evidence for this prediction. First, subjects were familiarized with a face with a name, say, Jim's face. Then they landed in one of three conditions. In the Studied Configuration condition, subjects were presented with two faces. One of the faces was Jim's original face and the other was Jim's face with a different nose. Subjects were then asked to identify which face was Jim's. The Transformed Configuration condition was just like the first except the face configurations were slightly altered, e.g., Jim's eyes were slightly further apart. In the Isolated Part Condition, subjects were asked to identify which of two facial features belonged to the familiarized face. For example, they were presented with Jim's mouth and John's mouth, and then had to identify which mouth belonged to Jim. Subjects were best at recognizing facial features in the Studied Configuration condition where the features were presented in the context of a whole face that had the same configuration as the familiarized face. This is what we should expect if face gestalts are perceptually experienced.

One takeaway from these studies is that visual experiences of gestalt properties are unique to face perception. This evidence, on its own, does nothing to support the idea that experts visually experience gestalt properties. But a growing consensus in the psychological literature is that humans are, in a sense, face experts, and perceptual expertise in general involves perceptually experiencing gestalt properties. ${ }^{13}$ There are a handful of studies I want to briefly go over that indicate experts display configural sensitivity with respect to objects of expertise, and thereby support the hypothesis that visual representations of gestalt properties figure into expert perception generally speaking.

Some of the most telling evidence in favor of the claim that visual expertise consists in visual experiences of gestalt properties comes from Gauthier and Tarr (I997). The authors created artificial stimuli known as 'Greebles', which are funny looking creatures that, like faces, have a small number of parts in a common configuration. Every Greeble belongs to one of five families and one of two genders. Subjects in their study were divided into two groups, either novices or experts. The experts were trained to recognize Greebles at the family, gender, and individual level over an average of 3240 trials.

In the testing phase, subjects were given a forced-choice task where a prompt specified one part of a specific Greeble ('Pimo's boges') followed by two pictures next to each other. Subjects were to choose which picture contained the target part of the target Greeble. There were three conditions. In the Studiedconfiguration condition, both pictures were of the target Greeble, one with the target part, and one with a foil part. The stimuli were the same in the

\footnotetext{
${ }^{13}$ Gauthier et al. 20Io provides a book-length analysis of this growing consensus.
} 
Transformed-configuration condition yet the top parts were moved I5 degrees to the front. In the Isolated-part condition, both pictures were of the target part and a foil part, yet were presented in isolation (see Figure 5). This study, in effect, matched the experimental design of Tanaka and Farah's whole-part tasks with faces.

Both experts and novices were better at recognizing the different parts of a Greeble when the part was presented in the context of a Greeble rather than in isolation. This finding mirrors the results rom Tanaka and Farah (I993). In addition, what is especially important is that subjects who underwent extensive Greeble training were more sensitive to subtle changes in Greeble configurations: the Greeble experts were better at recognizing all three different types of Greeble parts in the Studied-configuration than in the Transformedconfiguration. The Greeble experts did not display this configural sensitivity with inverted Greebles, and novices did not display such configural sensitivity. These results are exactly what we should expect to see if experts visually experience gestalt properties while novices do not. The Gauthier and Tarr (I997) results provide strong evidence that the representation of gestalt properties is not unique to face perception and recognition. Rather, the same mechanism that processes face configurations becomes reappropriated to process configurations that are relevant for a particular field of expertise.

Evidence on real world expertise points in the same direction. Let's revisit the Gobet and Simon studies on chess masters mentioned in the last section. The study directly pertains to how chess masters enjoy enhanced memory storage and retrieval capacities. But it indirectly shows show visual experiences of gestalt properties are helpful for chess masters. They have an enhanced ability to visually classify a particular chess board arrangement as check or checkmate. This would be mediated by visually experiencing the chess board arrangement as a unit, as a gestalt. Instead of having to check all of the relevant individual low-level features of the board, they only need to be aware of the pattern of those low-level features. Then this gestalt representation can be directly stored in memory. The Gobet and Simon studies don't show definitively that experts visually experience gestalts, but there are other studies that, when taken together with the research on expertise in lab settings, all suggest the same conclusion.

Inversion effects that are found with both faces and objects of expertise strengthen the case. Humans seem to have a special visual sensitivity to face stimuli presented in an upright orientation. A well-known demonstration is the Thatcher illusion (Thompson I980) (Figure 4). In the illusion, there is an image of Margeret Thatcher, but her eyes and mouth are flipped upside down. When this image is presented in an upright orientation, the image looks almost grotesque and we can easily detect the changes in the face. When the same altered image is presented in an inverted orientation, the image no longer looks grotesque and it is quite hard to detect abnormalities in the face. The 


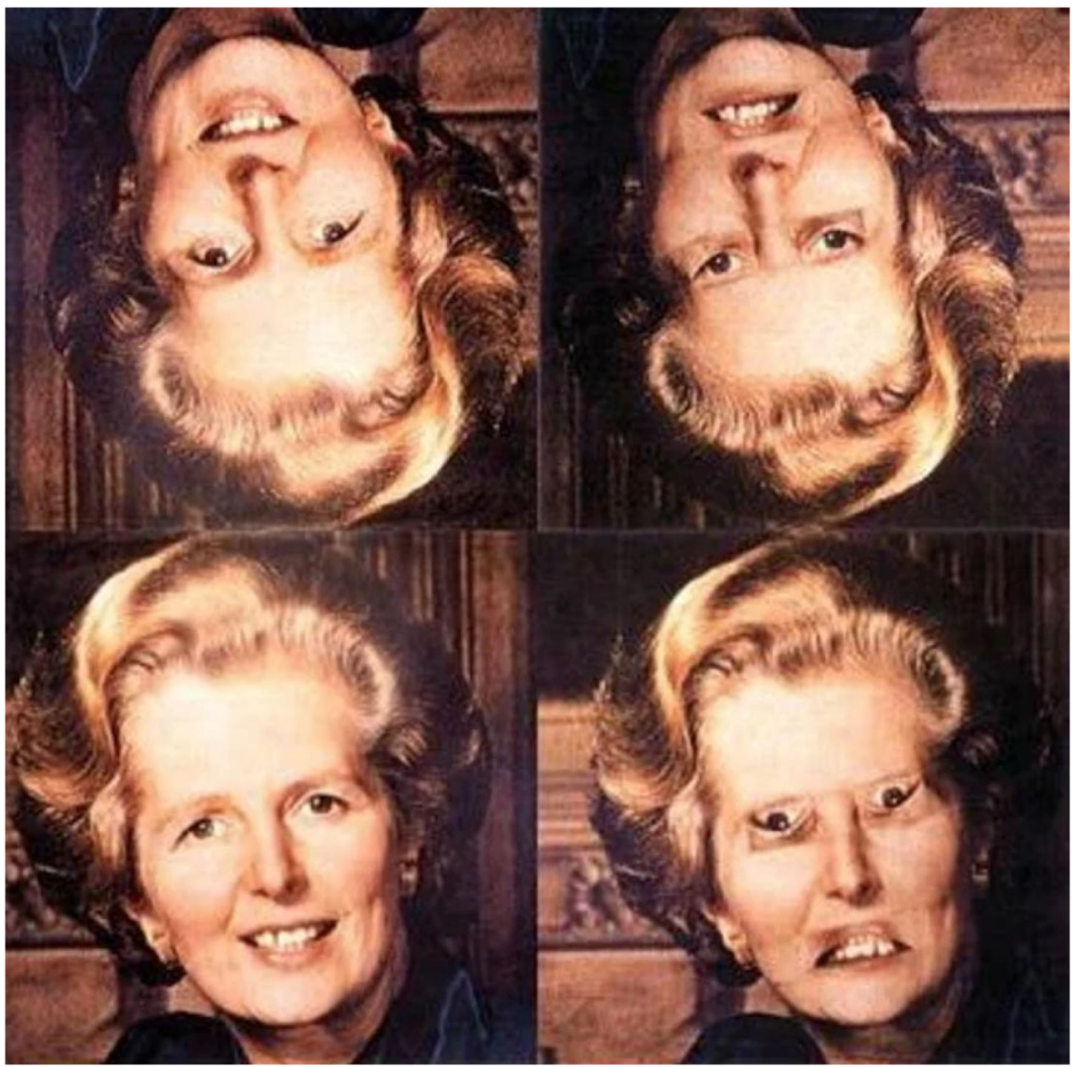

Figure 4. The Thatcher Effect. The bottom right photo appears almost grotesque. It is immediately obvious the face is distorted. When that same face is inverted, it is hard to tell if it is distorted at all.

altered upside down face looks the same as an unaltered upside down face. Therefore, low-level properties at different orientations can generate wildly different visual experiences and reactions. This is precisely what we would expect if we visually experience gestalt properties and have a special visual sensitivity to certain ones.

Inversion effects also occur for experts when experts are looking at some object of expertise. Diamond and Carey (I986) show that dog experts who can differentiate individual dogs of the same breed are also subject to inversion effects with images of dogs. Specifically, dog experts are worse at recognizing images of dogs they have previously seen when those images are presented in an inverted manner, whereas novices are equally good at recognizing images of dogs they've previously seen in both upright and inverted orientations. My account provides a simple, straightforward explanation of this data. In 
(a)

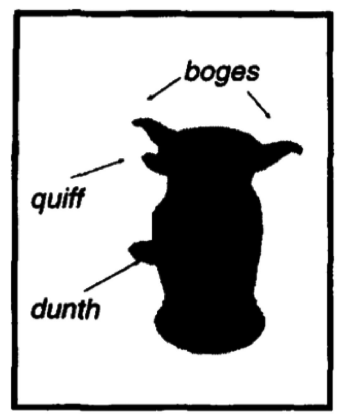

(b)
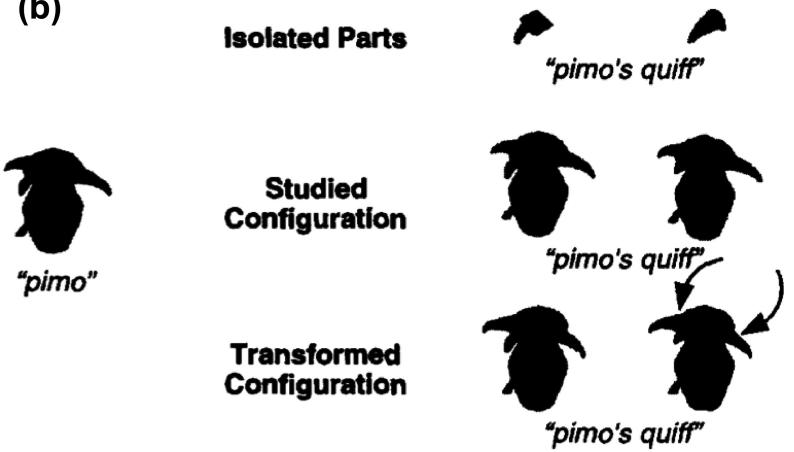

Figure 5. Greebles, the artificial stimuli created by and used in Gauthier and Tarr's (I997) study.

recognizing different breeds and individual dogs, dog experts rely on a representation of low-level properties in a particular configuration, i.e., a gestalt property. When the same low-level properties are inverted, the dog expert is looking at a new gestalt, because the relations between the low-level properties have changed in terms of which properties are above or below others. Since experts have become specifically tuned to certain gestalts (ones where the lowlevel properties are presented in an upright orientation), and since novices have not become specifically tuned to dog gestalts, the novices' recognitional abilities for inverted dog images are not as hampered as the dog experts' with the presentation of the inverted dog stimulus.

Research on radiologists and medical image perception further suggests that experts represent gestalt properties that novices do not. By representing gestalt properties rather than a set of low-level properties, radiologists more quickly and accurately identify abnormalities in medical scans. Indeed, multiple models of medical image perception-the holistic model (Kundel et al. (2007); the global-focal search model (Nodine and Kundel (1987); and the two-state detection model (Swensson (I980) - give a central role to expert 
visual representations of gestalt properties. The most telling evidence comes from eye-tracking studies on radiologists. Compared to novices, they spend less time processing each image, fixate on fewer locations, find abnormalities more quickly, and spend a longer time fixating on abnormalities. ${ }^{14}$ This data is nicely explained by my account. By visually representing gestalt properties, radiologists represent patterns or arrangements of the low-level properties of the image, and representing these patterns means radiologists need not check each individual low-level property to detect an abnormality.

In short, empirical data on face and expert recognition neatly fit with my account. The data ranges from expertise on artificial stimuli to expertise on chess, dogs, and medical images. While these studies do not directly rule out competing accounts, they significantly bolster the account that specialized visual experiences represent gestalt properties.

\section{IS THERE EMPIRICAL EVIDENCE AGAINST GESTALT?}

The reader may worry that there are other studies which seem to suggest that properties more distal and abstract than gestalts are represented in visual experience. The specific set of studies I have in mind are discussed by Block (20I4). In that paper, Block's main idea is to find some kind of effect that is unique to perception and then to show how the representation of certain properties are subject to that effect, such that we can conclude that those properties are perceptually represented.

Block focuses on adaptation effects found in some studies on emotional face perception. Adaptation effects take place when the presentation of some stimulus affects the experience of a subsequently presented stimulus. The waterfall illusion is an example of an adaptation effect. After staring at a waterfall, if one looks at a stationary object, the object will seem to be moving upwards. The explanation for this effect is that the threshold for the firing of neurons in the visual system is raised, such that the system becomes biased towards opposite stimuli. In the case of the waterfall illusion, the visual system becomes biased towards upward moving stimuli.

Block reasons that if we find perceptual adaptation effects, they can point us to what types of properties are perceptually represented. Butler et al. (2008) show that adaptation effects emerge with facial expressions of emotions. When focusing on a person's facial expression of anger, a subsequently presented neutral facial expression reportedly looks more scared than it would have if the neutral facial expression were focused on first (see Figure 6). The same goes for staring at a scared facial expression first: a subsequently presented

\footnotetext{
${ }^{14}$ This discussion of radiologists follows Sheridan and Reingold's (2017) review of holistic processing in medical image perception and Chudnoff's (2019) summary of their findings.
} 

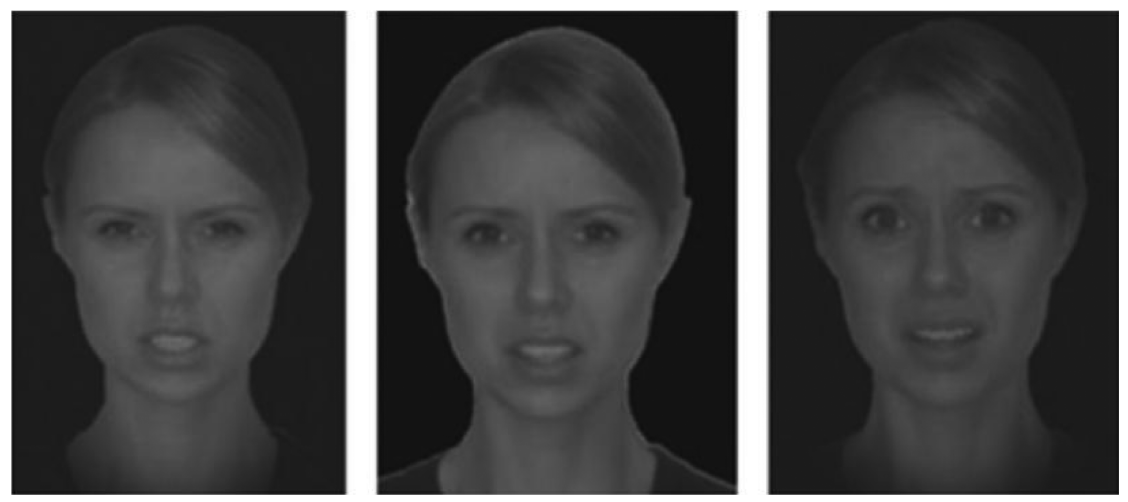

Figure 6. Facial expressions subject to perceptual adaptation. The middle facial expression will appear different depending on whether you stare intently at the facial expression on the right or left first. From Butler et al. (2008).

neutral facial expression seems angrier than it would have if the neutral facial expression were focused on first.

Assuming that adaptation effects only take place with perceptual representations, ${ }^{15}$ Butler et al.'s results suggest that whatever properties are subject to the effect are visually experienced. But does this adaptation finding tell us which properties are represented? Not quite. It could be that visually experiencing a gestalt property that is indicative of facial expressions of anger causes the subject to visually experience a subsequently presented neutral face as having a gestalt property that is indicative of a facial expression of fear. That is, we could also get perceptual adaptation effects with gestalt properties. What makes this gestalt interpretation plausible is that certain facial features stereotypically have different shapes and configurations that correspond to different facial expressions of emotions: the eyebrows furrow with expressions of anger, the eyes of fearful faces are more wide, and so on. In other words, we can tell what emotion is being expressed because we visually experience a gestalt property that is reliably indicative of that emotion. In short, then, Butler et al. (2008) fail to speak to which kind of property undergoes the perceptual adaptation effects, and so fails present evidence against Gestalt. ${ }^{16}$

${ }^{15}$ On this point, Helton (2016) argues that adaptation effects can take place with cognitive representations. If so, then showing that some representation is susceptible to adaptation effects does not yet show that it is perceptually represented.

${ }^{16}$ Block discusses another experiment on perceptual adaptation and faces by Susilo, McKone and Edwards (2010). Burge (20I4) (I think rightly) critiques Block's interpretation of the Susilo, McKone and Edwards (2010) experiment along the same lines as my critique of Butler et al. (2008), though he uses the terms 'generic' and 'specific' 'low-level attributives' rather than 'gestalt properties'. 
In general, I think it will be particularly tricky to design an empirical study that could rule out that gestalt properties are the contents of visual experience. There are two features of gestalt properties that problematize ruling them out this way. First, if it's true that members of a particular kind usually share a gestalt property, then it will be difficult to design a study where you can recognize a kind property without the gestalt property's presence. Second, while gestalt properties are abstracted away from some specific low-level properties, they can still be highly specific, e.g., face gestalts that correspond to different emotions can include the shapes of the arching of the eyebrows or the widening of the eyes. Any attempt to rule out one type of gestalt property would not rule out a more specific type of gestalt property. In short, perceptual adaptation studies do not suggest that we need to posit more than just gestalt properties and low-level properties as the contents of visual experience. Moreover, it will be difficult to design a study that could show this.

\section{TWO LINGERING POTENTIAL PROBLEMS}

Let me take stock. I've sketched an underexplored way of making sense of how Specialized could be true. The account I've sketched, Gestalt, is that specialized visual experiences represent gestalt properties through perceptual learning. I've laid out what I take gestalt properties to be and how perceptual learning could generate gestalt perception. I've also surveyed empirical evidence for the claim that gestalt properties are represented in expert visual experience. Then I looked at some empirical evidence that might be interpreted as a problem for my account, but I've shown how the results are what my account would predict.

At this point, I want to emphasize that the goal in this paper is to uplift the status of Gestalt as a main contender for making Specialized true, not necessarily to rule out all other possible contenders. Therefore, instead of focusing on every objection one could have to the many claims I've made throughout (e.g., about perceptual learning processes, about interpretations of empirical data, etc.), in what remains, I will only focus on two key objections to the basic claim that specialized visual experience represents gestalt properties rather than subordinate level rich properties. I will show how they can both be handled.

\section{VI.I The goldilocks problem}

One of the biggest problems facing any account that says gestalt properties are visually experienced is something I call the 'Goldilocks Problem'. The Goldilocks Problem is that gestalt properties have to be just the right level of abstractness to be able to make sense of how we recognize the things that we do: If gestalt properties are not abstract enough, or if they are too abstract, 
then gestalt properties won't be plausible candidates for the contents of visual experience. ${ }^{17}$

Look again at the Red-Bellied Woodpecker in Figure 2. A bird expert could quite effortlessly recognize it as a Red-Bellied Woodpecker, even though the bird expert has never seen any bird with all of those exact low-level properties in that exact configuration. Individual Red-Bellied Woodpeckers differ from one another in minor, detailed ways, much like humans differ from one another in minor, detailed ways. Few share the exact same shape, color, or pattern. How can the bird expert effortlessly recognize what kind of bird is in front of her if she visually experiences a specific arrangement of low-level properties that she has never visually experienced before?

We must be able to abstract away from the specific sizes, shapes, textures, and colors instantiated by objects and still classify the objects as sharing something similar based on some abstract invariant structure that is common to them all. We saw that gestalts are abstract, invariant, and determinable in Section II. These features do heavy lifting for Gestalt in response to this first part of the Goldilocks Problem. According to Gestalt, we can recognize different objects based on which gestalt properties we visually experience. There is some gestalt property that birds in general share, and there are different gestalts that indicate different kinds of birds. For example, the Red-Bellied Woodpecker gestalt property indicates Red-Bellied Woodpeckers; the Dove gestalt property indicates doves; and the Blue-Footed Booby gestalt indicates Blue-Footed Boobies. The bird expert doesn't visually experience these subordinate level natural kind properties, nor does she merely visually experience the very specific arrangement of low-level properties instantiated by a token Red-Bellied Woodpecker. She visually experiences the abstract, invariant, determinable gestalt property that Red-Bellied Woodpeckers have in common.

This seems a plausible way to address the first part of the Goldilocks Problem, but it makes way for the other part of the Goldilocks Problem to rear its head. Siegel (2010: III) most pressingly raises it. Once we isolate a gestalt property that is abstract enough to be common to all members of a particular kind, the worry is that that gestalt property will be abstract enough to be common to members of other kinds as well, in which case visually experiencing that gestalt property won't help us differentiate and recognize different kinds of things. Siegel writes about how at a certain level of abstraction, hair dryers and guns share the same shape gestalt, yet we can easily tell the difference between them, so it doesn't seem like that shape gestalt is what is visually experienced when we look at hair dryers and guns and tell them apart from each other. There is a simple response to this example, namely that we can point to details of the gun gestalt, like the shape of the trigger or its hammer,

\footnotetext{
${ }^{17}$ In case the reader is concerned about identifying a gestalt property for an extremely heterogeneous class, I address this closely related issue in 6.2 with the No Single Gestalt problem.
} 
and the larger circumference of the outlet of the hair dryer (the part where the air blows out), that differentiate the gun gestalt from the hair dryer gestalt. ${ }^{18}$

In order for the Goldilocks Problem to have weight, we need to find a real example where two kinds of things share all of the same gestalt and low-level properties, yet experts can still visually tell them apart. But it's quite difficult to find real life examples like this. Just as we did with the guns and hair dryers case, we can typically pinpoint some difference between the gestalts of two kinds of things, where the gestalt is just the right amount of abstraction to be diagnostic of a particular kind of rich property.

\section{VI.2 No single gestalt problem}

There is still a problem in the vicinity of the Goldilocks Problem. I call this the 'No Single Gestalt Problem'. The worry is that there cannot be just one abstract, invariant, determinable gestalt that is indicative of a kind of thing. For example, flamingos and ostriches are types of birds that seem like a counterexample to the claim that birds have the same gestalt in common. Flamingos and ostriches have particularly long necks and legs relative to the rest of their bodies, and other types of birds do not share these features. How could there be just one gestalt for all birds then?

There are three ways to respond. The first is to insist that birds really do share the same kind of abstract configuration. Going this route would involve spelling out what that abstract configuration is. To a first approximation, we can point to commonalities in the placement of various parts: the shape and placement of wings, eyes, legs, and beaks that all birds have in common. They also seem to share the same shapes in certain places, like curvature of where the back of the head meets the neck and back, and relatively thin legs. This suggests that birds plausibly have an abstract structure in common, even though the size or length of particular parts, like their legs or beaks, may vary widely across species.

The second way to respond is to agree that there is not one single abstract bird gestalt that is generally shared by all birds. There might be a couple of different bird gestalts-one that most kinds of birds share; another one that birds with extremely long necks, like flamingos and ostriches, share; and still another one that large-billed birds share. These three gestalts can still be indicative of the property of being a bird and associated with the concept BIRD, such that the visual experience of one of these gestalts still subserves bird recognition.

The third way to respond highlights the fact that experts make differentiations between members of the same basic kind, like birds, and categorize

\footnotetext{
${ }^{18}$ Alex Byrne in Siegel and Byrne (2016) discusses a similar case involving teacups and coffee cups. Byrne reaches the same conclusion as I, namely that there are ways in which the gestalts differ.
} 
them into even more specific, subordinate kinds, like Red-Bellied Woodpeckers. Members of subordinate kinds are more likely to share the same gestalt since there is typically less variance in members of subordinate kinds, e.g., Red-Bellied Woodpeckers generally look more like each other than they do Blue-Footed Boobies. That being said, it seems I can deflate the No Single Gestalt Problem by emphasizing the central claim that I'm making. The category of BIRDS is not a subordinate category-it's a basic category that both experts and novices can and do employ. In this paper, I am not proposing that gestalt properties, rather than basic level rich kinds, are visually experienced simpliciter. Rather, the proposal is that gestalt properties, rather than subordinate level rich kinds, are visually experienced by experts. So, the counterexample needs to be tweaked for it to have applicability. We would need to find an example where there is not a single gestalt that is indicative of a subordinate kind that experts recognize. Without such an example, however, this objection loses traction.

\section{GONCLUSION}

I've laid out and defended the Gestalt account, the heart of which is the claim that specialized visual experiences represent gestalt properties through perceptual learning. I've shown that the account has empirical and phenomenological merit. I've argued that objections to the claim that visual experiences represent gestalt properties rather than subordinate level rich properties can be straightforwardly addressed, especially once we have a particular conception of gestalt properties in mind. Still, as I've noted sporadically throughout the paper, my aim has been modest, to put Gestalt in the running, so to speak, as a good way of making sense of Specialized. I have not argued that it is the best way to make sense of Specialized. That is the aim for a future paper. ${ }^{19}$

\section{REFERENCES}

Arstila, V. (2016) 'Perceptual Learning Explains Two Candidates for Cognitive Penetration,' Erkenntnis, 81: $1 \mathrm{I} 5 \mathrm{I}-72$.

Bayne, T. (2009) 'Perception and the Reach of Phenomenal Content,' Philosophical Quarterly, 59: 385-404.

Block, N. (2014) 'Seeing-As in the Light of Vision Science,' Philosophy and Phenomenological Research, 89: $560-72$.

\footnotetext{
${ }^{19}$ For helpful discussion of these ideas, I am indebted to Magdalena Balcerak Jackson, Berit Brogaard, Adam Hauptfeld, Fiona Macpherson, Susanna Siegel, and the audiences at the $4^{\text {th }}$ iCog Conference, the 2018 Canadian Philosophical Association, and the 2019 Eastern APA. For detailed comments on earlier versions of this paper, thanks to Brendan Balcerak Jackson, Elijah Chudnoff, Kevin Connolly, David DiDomenico, and an anonymous reviewer for this journal.
} 
Burge, T. (2014) 'Reply to Block: Adaptation and the Upper Border of Perception,' Philosophy and Phenomenological Research, 89: 573-83.

Butler, A., Oruc, I., Fox, C. J. and Barton, J. J. (2008) 'Factors Contributing to the Adaptation Aftereffects of Facial Expression,' Brain Research, ingi: ir6-26.

Butterfill, S. (2009) 'Seeing Causings and Hearing Gestures,' Philosophical Quarterly, 59: 405-28. (20I5) 'Perceiving Expressions of Emotion: What Evidence could Bear on Questions about Perceptual Experience of Mental States?,' Consciousness and Cognition, 36: 438-51.

Chudnoff, E. (2019) 'Two Kinds of Cognitive Expertise,' Noûs, doi: Io.III I/nous.I2305.

Connolly, K. (2014) 'Perceptual Learning and the Contents of Perception,' Erkenn, 79: I407-18. (2017) 'Perceptual Learning,' in E. N. Zalta (ed.) The Stanford Encyclopedia of Philosophy. $<$ https://plato.stanford.edu/archives/sum2or7/entries/ perceptual-learning/ > accessed 9 November 2019.

(2019) Perceptual Learning: The Flexibility of the Senses. New York: Oxford University Press.

Diamond, R. and Carey, S. (I986) 'Why Faces Are and Are Not Special: An Effect of Expertise,' Journal of Experimental Psychology: General, II5: I07-17.

Firestone, C. and Scholl, B. J. (2016) 'Cognition Does Not Affect Perception: Evaluating the Evidence for "Top-down" Effects,' Behavioral and Brain Sciences, 39: I-72.

Fodor, J. (1998) Concepts: Where Cognitive Science Went Wrong. New York: Oxford University Press.

Gautier, I. and Tarr, M. (1997) 'Becoming a "Greeble" Expert: Exploring Mechanisms for Face Recognition,' Vision Research, 37: 1673-82.

Gibson, E. J. (I963) 'Perceptual Learning,' Annual Review of Psychology, I4: 29-56.

Gobet, F. and Simon, H. A. (1996) 'Templates in Chess Memory: A Mechanism for Recalling Several Boards,' Cognitive Psychology, 31: I-40.

(2000) 'Five Seconds or Sixty? Presentation Time in Expert Memory,' Cognitive Science, 24: $65 \mathrm{I}-82$.

Goldstone, R. and Byrge, L. (2015) 'Perceptual Learning,' in M. Matthen (ed.) The Oxford Handbook of Philosophy of Perception. New York: Oxford University Press.

Helton, G. (2016) 'Recent Issues in High-level Perception,' Philosophy Compass, II: 851-62. (2018) 'Visually Perceiving the Intentions of Others,' Philosophical Quarterly, 68: 243-64.

Kundel, H. L., Nodine, C. F., Conant, E. F. and Weinstein, S. P. (2007) 'Holistic Component of Image Perception in Mammogram Interpretation: Gaze-tracking Study,' Radiology, 242: $396-402$.

Logue, H. (2013) 'Visual Experience of Natural Kind Properties: Is There Any Fact of the Matter?' Philos Stud, I62: I-I2.

Macpherson, F. (2012) 'Cognitive Penetration of Colour Experience: Rethinking the Issue in Light of an Indirect Mechanism,' Philosophy and Phenomenological Research, 84: 24-62.

Mandelbaum, E. (2018) 'Seeing and Conceptualizing: Modularity and the Shallow Contents of Perception,' Philosophy and Phenomenological Research, 97: 267-83.

Neufeld, E. (2018) 'Can We Perceive Mental States?' Synthese. https://doi.org/Io.1007/ sII229-0I8-I807-7, accessed 9 November 2019.

Nodine, C. F. and Kundel, H. L. (I987) 'The Cognitive Side of Visual Search in Radiology,' in J. K. O'Regan and A. Levy-Schoen (eds) Eye Movements: From Physiology to Cognition, 573-82. Amsterdam: Elsevier.

Sheridan, H. and Reingold, E. M. (2017) 'The Holistic Processing Account of Visual Expertise in Medical Image Perception: A Review,' Frontiers in Psychology, 8: I620.

Siegel, S. (2006) 'Which Properties Are Represented in Perception?,' in T. M. Gendler and J. Hawthorne (eds) Perceptual Experience. New York: Oxford University Press.

_ (2009) 'The Visual Experience of Causation,' Philosophical Quarterly, 59: 519-40.

(2010) The Contents of Visual Experience. New York: Oxford University Press.

(2014) 'Affordances and the Contents of Perception,' in B Brogaard (ed.) Does Perception Have Content?

Byrne, A. (2016) 'Rich or Thin?,' in B. Nanay (ed.) Current Controversies in the Philosophy of Perception. London: Routledge.

Routledge.

Siewert, C. (1998) The Significance of Consciousness. Princeton: Princeton University Press.

Silins, N. (201 I) 'Seeing Through the 'Veil of Perception', Mind, I20: 329-67. 
Stokes, D. (20I4) 'Cognitive Penetration and the Perception of Art,' Dialectica, 68: I-34. (2018) 'Rich Perceptual Content and Aesthetic Properties,' in A. Bergqvist and R. Cowan (eds) Evaluative Perception, I9-4I. Oxford University Press.

Susilo, T., McKone, E. and Edwards, M. (2010) 'Solving the Upside-down Puzzle: Why do Upright and Inverted Face Aftereffects Look Alike?' fournal of Vision, Io: I.

Swensson, R. G. (I980) 'A Two-stage Detection Model Applied to Skilled Visual Search by Radiologists,' Perception E Psychophysics, 27: I I-6.

Tanaka, J. W. and Farah, M.J. (I993) 'Parts and Wholes in Face Recognition,' The Quarterly Journal of Experimental Psychology Section A, 46A: 225-45.

Thompson, P. (I980) 'Margaret Thatcher: A New Illusion,' Perception, 9: 483-4.

Toribio, J. (2018) 'Visual Experience: Rich but Impenetrable,' Synthese, i95: 3389-406.

Young, A. W., Hellawell, D. and Hay, D. C. (I987) 'Configurational Information in Face Perception,' Perception, I6: 747-59.

University of Miami, USA 